\title{
Directory of Culturally Competent Health Care Providers for Gender \& Sexual Minorities
}

\author{
Wilhelmina Nicholson
}

(C) Springer Science+Business Media, LLC 2010

People in the LGBTQI and alternative sexuality and relationship communities, like most other people, face great difficulty accessing affordable, comprehensive health care. Systemic discrimination and lack of basic cultural competency prevents many people from accessing medically necessary and appropriate health care and social services. In an attempt to ensure access to safe, respectful and non-discriminatory health care for the LGBTQI and Sex-Positive communities (and the diverse subcommunities within them), I wish to create a quality-controlled directory of health care providers willing and able to provide quality, culturally competent care to gender and sexual minorities. Unlike existing lists serving the various communities separately, this list will allow providers to designate areas of gender and sexuality experience and competence so that patients can select providers that meet their individual needs!

If you would like to be added to our list, please submit your entries using this quick and easy survey: http://www.surveymonkey.com/s/JudgmentFreeHealthCare Providers.

W. Nicholson $(\bowtie)$

B.S./M.S. Dual-Degree Candidate in Nurse Midwifery,

College of Nursing, New York University, New York, NY, USA

e-mail: Mina.Nicholson@nyu.edu 